\section{LAB-FORTH software package}

\author{
FRODE SVARTDAL \\ University of Tromso, Tromso, Norway
}

It is well documented that FORTH is an outstanding software tool in laboratory computer control systems (Berg \& Kaiser, 1985; Lea, 1982; Marshall-Goodell, Gormezano, Scandrett, \& Cacioppo, 1981; Orr, 1984; Scandrett \& Gormezano, 1980). Among the attractive features of FORTH are the following.

First, FORTH is fast and is well suited for real-time control applications. If further speed is required, timecritical routines may be written (or rewritten) in machine code by means of the FORTH Assembler found in most FORTHs. Second, FORTH is compact, requiring little computer memory. FORTH is a compiled language. Code is compiled by threading together previously compiled routines. A routine, or "word" in FORTH, is executed by calling a sequence of previously defined words (routines). An advantage of this method of compilation is that the language occupies very little computer space. Another advantage is that the language is flexible and extensible. At the outset, the language consists of a set of fundamental routines. New routines are based on this nucleus. As new routines are added, the language itself grows.

Third, once the user is acquainted to reverse Polish notation and use of the stack, FORTH may be viewed as almost ideal as a user interface to the computer. FORTH is an interactive language, and the user may test routines or parts of routines as soon as they are written. Specific tasks to be performed may be defined and given names according to functions. If good names are chosen, programs are not only self-documenting but also intuitively understandable. Furthermore, FORTH programming encourages simple and logical thinking (see Brodie, 1984).

In spite of the obvious advantages of FORTH, the availability of reasonably priced laboratory control software packages written in FORTH is limited. LAB-FORTH was developed in our laboratory to control external equipment from a standard Apple IIe microcomputer. Given the routines contained in LAB-FORTH, even novice computer users should be able to write application programs with only a minimum of training.

Description. LAB-FORTH is written in MasterFORTH (Apple II Version 1.1), and requires an interface using the 6522 VIA. The present version features eight input and eight output channels, but may be easily expanded. A number of commonly used routines, such as input, output, and counting routines, are provided. They are all

The author's mailing address is: Institute of Social Science, University of Tromso, P. O. Box 1040, 9001 Tromso, Norway. written in machine code for maximum speed. Application programs are based on these routines, and may be written in FORTH or assembly language.

Basic Routines. Extremely simple control of output signals is possible in LAB-FORTH. To turn on a given output channel, the relevant output routine is invoked by executing the relevant LAB-FORTH "word." For example, to turn on an output channel, the word " $\mathrm{ON}$ " is executed. To turn it off, the word "OFF" is executed. Since these words expect a value to operate on, the relevant channel number must be put on the stack before "ON" and "OFF" are executed. To turn on channel 1, simply write "CH1 ON." To turn off channel 1, write "CH1 OFF."

Because FORTH is extensible, new routines (words) are easily implemented. For example, to pulse channel 1 , the words "ON" and "OFF" may be combined into a new word called "CH1PULS." A possible FORTH definition (which starts with a colon and ends with a semicolon) of this new word is

\section{: CH1PULS CH1 OFF CH1 ON CH1 OFF ;}

If output channels are regularly used to control specific environmental events, output words may be renamed in order to facilitate programming. For example, if channel 1 is used to control a lamp, " $\mathrm{CHl}$ " may be renamed "LIGHT." To turn on the light, "LIGHT ON" is executed; to turn it off, "LIGHT OFF" is executed. Renaming may be performed in the assembler source code or in FORTH.

Several input routines are available. For example, the word "AIN" reads the 6522 input register to check whether any input lines are active. The words "CNTn+" (where $n$ may be a value between 1 and 8) increment a cumulative counter related to the relevant input or output channel. Such counters, which use variables defined in FORTH, may be accessed both in FORTH and assembler definitions.

An interrupt routine is provided, permitting an external signal to trigger specific routines. Interrupt operation is initialized by the word "IRQON" and turned off by the word "IRQOFF." The routine performed during an interrupt must be written by the user to suit specific needs, but the general interrupt handling routine is provided.

Application Programs. Control of external events, counting, timing, and decisions may easily be performed using the fundamental routines. Data may be stored and written to diskette. A few application programs are provided, but the user should be able to write new application programs with a minimum of training using the predefined words.

Conclusion. The fundamental routines provided in LAB-FORTH may be used to construct powerful and very fast laboratory control programs. Comparable programs written in BASIC, even if these are compiled or make frequent calls to machine code subroutines, are not nearly 
as efficient and fast as the FORTH version. If necessary, FORTH words may easily be rewritten in machine code for greater speed. Additional FORTH advantages are its clean and modular routines and extremely compact code.

Availability. A 30-page manual describing the features of LAB-FORTH may be obtained from the author. Requests should include $\$ 5$ to cover postage and reproduction costs.

\section{REFERENCES}

BERG, K. P., \& KaISER, R. E. (1985). Stepper motor actuation by FORTH. Intelligent Instruments and Computers, 3, 11-15.
Brodie, L. (1984). Thinking FORTH. Englewood Cliffs, NJ: Prentice-Hall.

LEA, D. (1982). Using the FORTH language in real-time computer applications. Behavior Research Methods \& Instrumentation, 14, $29-31$.

ORR, J. L. (1984). Going FORTH in the laboratory. Behavior Research Methods, Instruments, \& Computers, 16, 193-198.

Marshall-Goodell, B., Gormezano, I., SCANdrett, J., \& Cacioppo, J. T. (1981). The microcomputer in social-psychological research: An Apple II/FIRST laboratory. Sociological Methods \& Research, 9, 502-512.

Scandrett, J., \& Gormezano, I. (1980). Microprocessor control and A/D data acquisition in classical conditioning. Behavior Research Methods \& Instrumentation, 12, 120-125.

(Manuscript received for publication October 25, 1985.) 